\title{
COGNITIVE ABILITIES RELATED TO DRIVING PERFORMANCE IN A SIMULATOR AND CRASHING ON THE ROAD
}

\author{
Steven W. Anderson ${ }^{1}$, Matthew Rizzo ${ }^{1}$, Qian Shi $^{2}$, \\ Ergun Y. Uc ${ }^{1}$, and Jeffrey D. Dawson ${ }^{2}$ \\ ${ }^{1}$ Department of Neurology \\ ${ }^{2}$ Department of Biostatistics \\ University of Iowa \\ Iowa City, Iowa, USA \\ E-mail: steven-anderson@uiowa.edu
}

\begin{abstract}
Summary: The purpose of this study was to examine the relationships between performances on standardized neuropsychological measures of cognitive abilities, simulated driving performance, and state crash records in drivers with cognitive decline due to aging and dementia. Participants were 202 experienced older adult drivers ages 55 years and older: 70 had mild dementia due to probable early Alzheimer's disease and 132 had no neurologic disease. All completed a battery of neuropsychological tests and drove contemporaneously on a high fidelity simulator. The participants' State Department of Transportation driving records were monitored for up to two years after testing. The simulator composite score, reflecting overall driving ability, was significantly correlated with overall cognitive ability, as indexed by the neuropsychology composite score, as well as with individual cognitive tests of attention, memory, visuospatial and visuomotor abilities. Drivers who crashed during an intersection incursion scenario performed significantly worse on the composite measure of cognitive function than those who successfully steered around the incurring vehicle. Crashers had specific cognitive deficits on measures of visuomotor abilities and attention. Memory test performances for both verbal information and visual material were associated with subsequent on-road crashes. These findings provide support for the validity of driving simulation as a safe means of evaluating a range of driving responses that cannot be tested on the road, and suggest that relatively simple and inexpensive neuropsychological tests of specific cognitive abilities could be used to help evaluate older drivers' risk of unsafe driving
\end{abstract}

\section{INTRODUCTION}

Driving an automobile requires a seamless interaction of multiple cognitive abilities, including attention, perceptuomotor skills, memory, and decision-making. Because these various cognitive functions are processed in partially segregated neural systems, damage to the brain can result in various profiles of impaired and preserved cognitive abilities. Analysis of the effects on driving of impairments in specific domains of cognition is a potentially fruitful approach to developing better models of driving and facilitating evaluation of driving competency.

When drivers have impairments of cognition resulting from brain dysfunction, the risk of safety errors generally increases (e.g., Galski et al., 1993; Reger et al., 2004). It is likely that deficits in different aspects of cognition have different implications for driving ability, but there has been limited study to date of the relationships between specific cognitive abilities and driving 
behaviors. Much of the relevant research has focused on visual processing speed and attention, and deficits in this domain have been related to increased crash risk (e.g., Ball and Owsley, 1993; Ball et al., 1993; Duchek et al., 1998; Parasuraman and Nestor, 1991). There also has been some study of the effects of impairments of memory on driving. Difficulties with episodic memory are common in many neurologic conditions, and may dissociate from relatively preserved procedural or motor memory. Such deficits appear to impact most strongly on certain aspects of driving, such as route-following, while preserved procedural memory abilities allow for generally good vehicle control (Anderson et al., 2005; Cavaco et al., 2004; Uc et al., 2004).

As our population ages, concern is growing about the safety implications of the increasing number of elderly drivers, and particularly those with age-associated cognitive decline or dementia. It is known that patients with Alzheimer's disease (AD) become unsafe drivers (e.g., Friedland et al., 1988; Rizzo et al., 2001; Rizzo et al., 1997; Tuokko et al., 1995). However, this diagnosis alone is not sufficient grounds for restricting driving privileges, as some patients in the early stages of this progressive condition may have only isolated memory deficits and may maintain driving ability comparable to their non-dementing peers.

There is a need for better understanding of the relationships between cognitive impairments and driving abilities in older adults. The purpose of this study was to examine the relationships between performances on standardized neuropsychological measures of cognitive abilities and driving performance in a group of older adults with and without neurologic disease. Elucidation of relationships between specific cognitive abilities and driving performance in older adults will help identify the critical cognitive factors that contribute to poor driving in old age, and provide guidance for the evaluation of older individuals for fitness to drive.

\section{METHODS}

\section{Participants}

Two hundred two drivers over the age of 55 participated in this study, including 132 subjects without neurologic disease and 70 subjects with mild dementia due to probable early AD. Participants had an average age of 71.1 years (standard deviation=7.6), with slightly more males than females (55\% vs. $45 \%$ ). Years of education ranged from 8 to 20 with a mean (standard deviation) of $15.3(2.8)$.

\section{Procedures}

All participants completed a battery of neuropsychological tests and contemporaneously took a drive on a high fidelity simulator (four channel, 150-degree forward view, and 50-degree rear view; $60-\mathrm{Hz}$ visual update rate in a dynamic driving scenario with multiple interacting vehicles). In addition, their State Department of Transportation driving records for subsequent years were monitored for up to two years.

Neuropsychological Evaluation. The neuropsychological tests included standardized measures of visuospatial and visuomotor abilities, verbal and visual memory, attention, language, and executive functions, and a composite score was generated based on the combined performance across all neuropsychological measures. The battery included the following tests: anterograde 
verbal memory (Rey Auditory Verbal Learning Test, AVLT; Lezak, 1995), visual memory for geometric designs (Benton Visual Retention Test, BVRT, Sivan, 1992, and 30 minute delayed recall of the Complex Figure Test, CFT Recall, Lezak, 1995), executive functions, i.e., attention and working memory (Trailmaking Test, TMT, Heaton et al., 1991), spatial perception (Judgment of Line Orientation, JLO, Benton et al., 1983), language (Controlled Oral Word Association, COWA, Benton et al., 1994) and visuomotor abilities (Complex Figure Test, CFT, Lezak, 1995; WAIS-III Block Design, Wechsler, 1997).

Driving Simulation. Participants were tested using driving simulation scenarios implemented on the Simulator for Interdisciplinary Research in Ergonomics and Neuroscience (SIREN). SIREN is a four-channel, 150-degree forward view, and 50-degree rear view high performance driving simulator. The simulator cab is a four-door 1994 GM Saturn SL2. Simulation software was provided by Drive Safety (Fort Collins, CO). Four PC image generators created the graphics for each of the four visual channels. A separate PC host computer controlled the general operation of the simulator and another PC provided the platform to design scenarios, run simulations and collect data. Accelerator position, speed, brake pedal position, and steering wheel position were continuously monitored. SIREN maintained a $60-\mathrm{Hz}$ visual update rate in a dynamic driving scenario with multiple interacting vehicles. Four Epson 710C polysilicon extended high aperture LCD projectors with 1024 x 768 resolution and 1000ANSI (American National Standards Institute) lumen brightness display real-time graphics at $60 \mathrm{~Hz}$. All street signs and roads conformed to the requirements of AASHTO (American Association of State Highway and Transportation Officials) and MUTCD (Manual for Uniform Traffic Control Devices). A tilebased scenario development tool allowed us to select from different road types and populate roadways with different vehicles that interacted with drivers and each other.

A research assistant familiarized participants with the vehicle controls. Microphones in the car allowed an operator in the control area located adjacent to the simulator to monitor onboard activity and start and stop the simulation on demand. Participants drove on a simulated rural 2lane highway with interactive traffic resembling a drive on the roads of Iowa City, Iowa, and similar to the roads most participants would travel regularly. Participants were asked to drive as if driving in the real world. The experimental drive began with several miles of uneventful driving on a rural highway with a speed limit of $55 \mathrm{mph}$. Events experienced during the drive included encountering a lead vehicle stopped at a 4-way intersection waiting to turn left, and a traffic light triggered to turn yellow when the participant vehicle was 2.4 seconds from the intersection (requiring a rapid go-no go decision, with sufficient time to stop safely).

At the final intersection (after a segment of uneventful highway), participants encountered a crash avoidance challenge. In this scenario, approach by the participant to within 4.0 seconds of the intersection triggered an illegal incursion by another vehicle entering the intersection from the driver's right side. The primary dependent measure was the occurrence or avoidance of a collision with the incurring vehicle, and additional analyses were performed on crash severity and crash avoidance behaviors (deceleration, time to braking, steering maneuvers).

Driving performance was measured throughout the drive, using steering wheel position variability, normalized braking and accelerator positions, and speed. The videotapes from the SIREN drives were reviewed for detailed data reduction and on-road safety errors. Straight, uneventful segments of the SIREN drive were used in the analysis of speed and steering control. 
In addition to the crash avoidance challenge explained above, the simulated drive included several other standardized challenges. A composite index of driving ability was calculated from standardized t-scores (mean $=50, \mathrm{SD}=10$ ) from nine different driving and task outcomes (awareness of current speed, awareness of a following vehicle, ability to multi-task, intersection incursion result, left-turning vehicle result, response to an emergency vehicle, at-fault safety errors, speed control, and steering control), with higher scores reflecting better drivers.

\section{RESULTS}

Overall cognitive ability, as indexed by the neuropsychology composite score, was significantly correlated with the simulator composite score reflecting overall driving ability (Spearman $\mathrm{r}=$ 0.34; $\mathrm{p}<0.001)$. All of the individual cognitive tests, with the exception of COWA, were significantly correlated with the simulator composite score (see Table 1).

Table 1.Spearman correlations between cognitive tests and the simulator composite score

\begin{tabular}{|l|c|c|}
\hline Test & Correlation & P-value \\
\hline AVLT & $28 \%$ & $<0.001$ \\
BVRT (errors) & $-24 \%$ & 0.007 \\
CFT-Recall & $20 \%$ & 0.026 \\
Trails-A (time) & $-23 \%$ & 0.007 \\
Trails-B (time) & $-28 \%$ & 0.002 \\
JLO & $39 \%$ & $<0.001$ \\
CFT-Copy & $27 \%$ & 0.002 \\
Block Design & $32 \%$ & $<.001$ \\
COWA & $14 \%$ & 0.105 \\
\hline
\end{tabular}

Drivers who crashed during the intersection incursion scenario performed significantly worse on the composite measure of cognitive function than those who successfully steered around the incurring vehicle (mean scores of 354 vs. 403; $p=0.001$ ). Crashers had specific cognitive deficits on measures of visuomotor abilities (CFT Copy, $\mathrm{p}=0.002$ and WAIS-III Block Design, $\mathrm{p}=0.003$ ) and executive functions (TMT Part $\mathrm{B}, \mathrm{p}=0.001$ ). Memory test performances, for both verbal information (Rey AVLT, $\mathrm{p}=0.004$ ) and visual material (CFT Recall, $\mathrm{p}=0.036$ ), were related to subsequent on-road crashes (see Figures 1 and 2).

\section{CONCLUSIONS}

These findings indicate that cognitive abilities in older adults, as measured by standardized neuropsychological tests, are associated with driving performance on a simulator and with realworld crashes. An index of overall cognitive ability, which summarized performances across multiple cognitive domains, predicted general driving competency on the simulator, as well as crashes on the simulator. Weaknesses in certain cognitive abilities, including visuomotor abilities, executive functions, and memory, were particularly associated with poorer driving and increased the risk of crashing. 


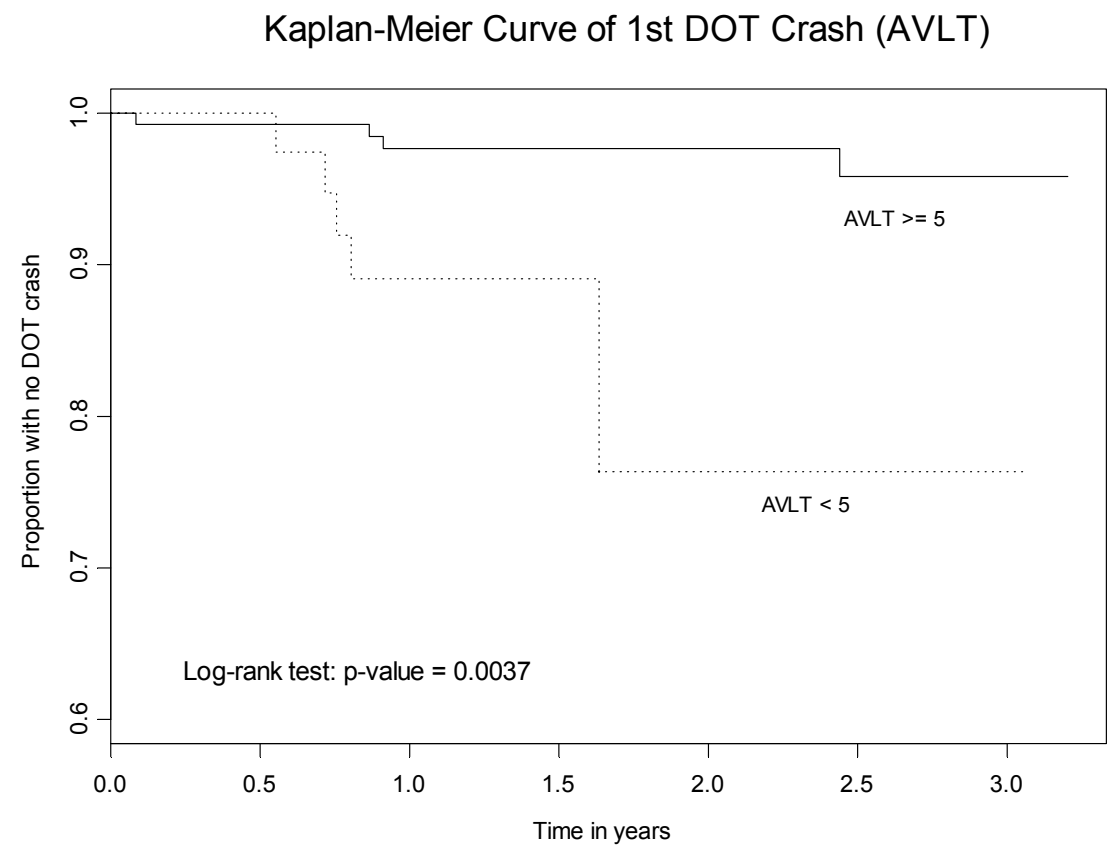

Figure 1. The estimated survival curves for the time-until-DOT-crash, for two categories of AVLT values

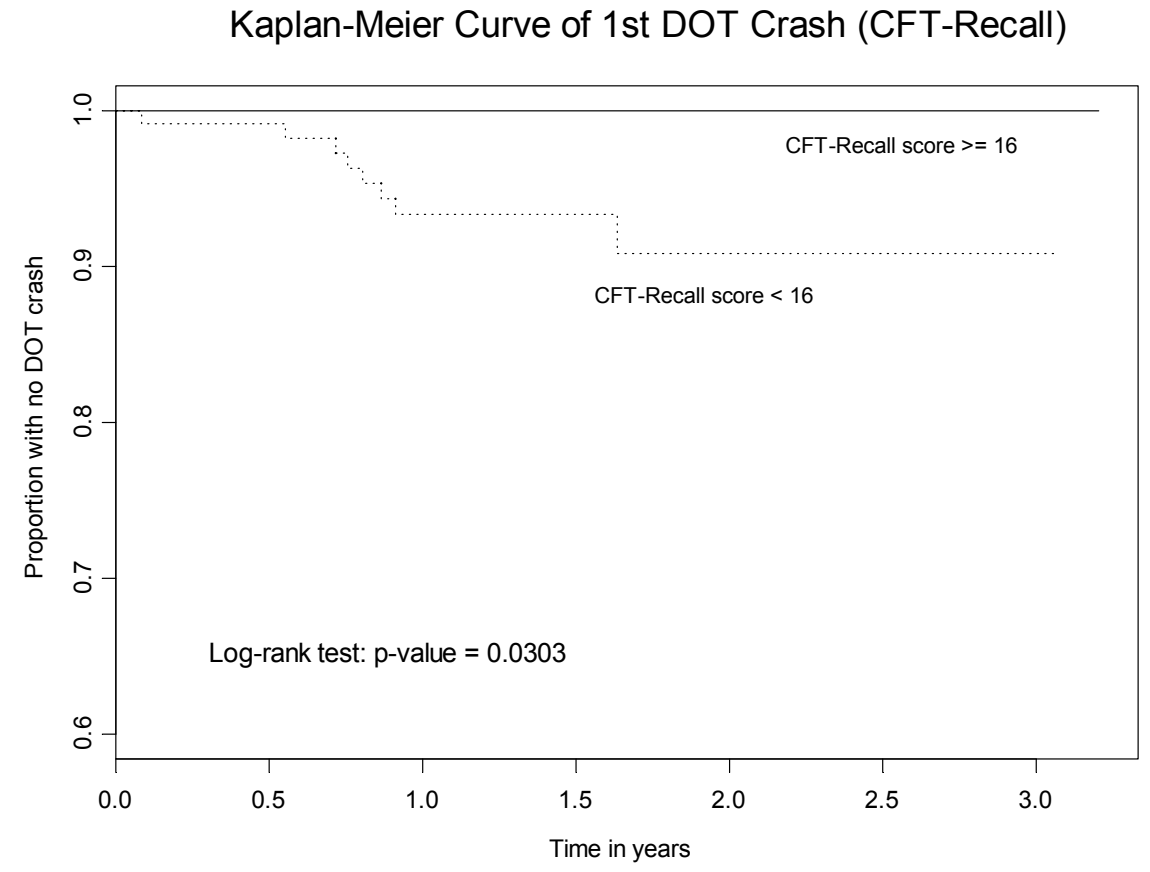

Figure 2. The curves according to CFT-Recall score

These findings provide support for the validity of driving simulation as a safe means of evaluating a range of driving responses that cannot be tested on the road, and suggest that 
relatively simple and inexpensive neuropsychological tests of specific cognitive abilities could be used to help evaluate older drivers' risk of unsafe driving.

The simulator allowed evaluation of the older drivers' responses to a potentially dangerous situation, providing information regarding safety risk that cannot readily be obtained by other means. Consideration of responses to such situations may be particularly important for evaluating driving safety in persons with early Alzheimer's disease and related conditions, because these individuals often can perform adequately in familiar and routine situations, but become confused in unexpected or unfamiliar conditions. Complementary information was obtained from the simulator composite score, which provided an index of overall driving competency across multiple domains of driving performance over the course of the drive. The value of this index is in monitoring the cumulative frequency of relatively minor (i.e., non-crash) driver errors that increase the probability of crashing. We are continuing to evaluate the concurrent and predictive validity of these simulator performance measures through comparison to driving performance in an instrumented on-road vehicle and ongoing monitoring of State driving records.

The findings from this study and others (see Reger, 2004, for a review) make clear that deficits in multiple aspects of cognition can contribute to unsafe driving. It is thus unlikely that any single neuropsychological test will be adequate for screening of cognitive deficits relevant to driving. Rather, a brief battery of tests that provides coverage of the key domains of cognition necessary for safe driving appears to be necessary. Specific domains of cognition that should be considered include attention, memory, spatial perception, and visuomotor coordination. Because normal aging and age-related neurodegenerative conditions such as Alzheimer's disease affect multiple interacting aspects of cognition, we have been evaluating the validity of a composite index reflecting overall cognitive ability for the prediction of driver safety. The findings of this study and others (Rizzo et al., 2001; Uc et al., 2004) provide support for this approach. We anticipate that continued research in this vein will lead to a brief (less than 15 minutes), standardized, inexpensive cognitive screening battery, with automated scoring algorithms and empirically-derived cutoff scores, that will provide a fair and efficacious means of determining driver safety.

\section{ACKNOWLEDGMENTS}

This study was supported by NIA AG 15071 .

\section{REFERENCES}

Anderson, S.W., Rizzo, M., Skaar, N., Stierman, L., Cavaco, S., Dawson, J., and Damasio, H. (In press). Amnesia and driving. Journal of Clinical and Experimental Neuropsychology.

Ball, K. and Owsley, C. (1993). The Useful Field of View Test: A new technique for evaluating age-related declines in visual function. Journal of the American Optometric Association, 64: 71-79.

Ball, K., Owsley, C., Sloane, M..E., Roenker, D.L. and Bruni, J.R. (1993). Visual attention problems as a predictor of vehicle crashes in older drivers. Investigative Ophthalmology and Visual Science, 34: 3110-3123. 
Cavaco, S., Anderson, S.W., Allen, J.S., Castro-Caldas, A.L. and Damasio, H. (2004). The scope of preserved procedural memory in amnesia. Brain, 127: 1853-1867.

Duckek, J.M., Hunt, L., Ball, K., Buckles, V., and Morris, J.C. (1998). Attention and driving performance in Alzheimer's disease. Journal of Gerontology: Psychological Sciences, 53B: P130-P141.

Friedland, R.P., Koss, E., Kumar, A., Gaine, S., Metzler, D., Haxby, J.V., and Moore, A. (1988). Motor vehicle crashes in dementia of the Alzheimer type. Annals of Neurology, 24: 782-786.

Galski, T., Bruno, R.L. and Ehle, H.T. (1993). Prediction of behind-the-wheel driving performance in patients with cerebral brain damage: A discriminant function analysis. American Journal of Occupational Therapy, 47: 391-396.

Parasuraman, R. and Nestor, P.G. (1991). Attention and driving skills in aging and Alzheimer's disease. Human Factors, 33: 539-557.

Reger, M.A., Welsh, R.K., Watson, G.S., Cholerton, B., Baker, L.D. and Craft, S. (2004). The relationship between neuropsychological functioning and driving ability in dementia: A metaanalysis. Neuropsychology, 18: 85-93.

Rizzo, M., McGehee, D., Dawson, J., and Anderson, S.W. (2001). Simulated car crashes at intersections in drivers with Alzheimer's disease. Alzheimer Disease and Associated Disorders, 15: $10-20$.

Rizzo M., Reinach, S., McGehee, D., Dawson, J. (1997). Simulated car crashes and crash predictors in drivers with Alzheimer's disease. Archives of Neurology, 54: 545-553.

Tuokko, H., Tallman, K., Beattie, B.L., Cooper, P. and Weir, J. (1995). An examination of driving records in a dementia clinic Journal of Gerontology: Social Sciences, 50B: S173S181.

Uc, E.Y., Rizzo, M., Anderson, S.W., Shi, Q. and Dawson, J.D. (2004). Driver route-following and safety errors in early Alzheimer's disease. Neurology, 63: 832-837. 